Proceedings of the Edinburgh Mathematical Society (2003) 46, 545-559 (C)

DOI:10.1017/S0013091503000026 Printed in the United Kingdom

\title{
SELF-INTERSECTIONS OF SURFACES AND WHITNEY STRATIFICATIONS
}

\author{
RÜDIGER ACHILLES AND MIRELLA MANARESI \\ Dipartimento di Matematica, Università di Bologna, \\ Piazza di Porta S. Donato 5, I-40126 Bologna, \\ Italy (achilles@dm.unibo.it; manaresi@dm.unibo.it)
}

(Received 6 January 2003)

\begin{abstract}
Let $X$ be a surface in $\mathbb{C}^{n}$ or $\mathbb{P}^{n}$ and let $C_{X}(X \times X)$ be the normal cone to $X$ in $X \times X$ (diagonally embedded). For a point $x \in X$, denote by $g(x):=e_{x}\left(C_{X}(X \times X)\right)$ the multiplicity of $C_{X}(X \times X)$ at $x$. It is a former result of the authors that $g(x)$ is the degree at $x$ of the Stückrad-Vogel cycle $v(X, X)=\sum_{C} j(X, X ; C)[C]$ of the self-intersection of $X$, that is, $g(x)=\sum_{C} j(X, X ; C) e_{x}(C)$. We prove that the stratification of $X$ by the multiplicity $g(x)$ is a Whitney stratification, the canonical one if $n=3$. The corresponding result for hypersurfaces in $\mathbb{A}^{n}$ or $\mathbb{P}^{n}$, diagonally embedded in a multiple product with itself, was conjectured by van Gastel. This is also discussed, but remains open.
\end{abstract}

Keywords: hypersurface singularities; normal cone; Whitney stratification

2000 Mathematics subject classification: Primary 32S15

Secondary $13 \mathrm{H} 15 ; 14 \mathrm{C} 17$

\section{Introduction}

In his article [20], Whitney introduced the notion of a regular stratification (later called Whitney stratification) that turned out to be a very useful tool in the study of singular complex analytic spaces. Whitney's regularity condition had been characterized numerically by Teissier [17] using polar multiplicities. We will use Teissier's numerical criterion (see $\S 2$ ) and the algebraic approach to intersection theory by Stückrad and Vogel (see $\S 3$ ) to construct the canonical (or minimal) Whitney stratification of a surface $X$ in $\mathbb{P}^{3}$. More precisely, let $C_{X}(X \times X)$ be the normal cone to $X$ in $X \times X$ (diagonally embedded). For a point $x \in X$, denote by $g(x):=e_{x}\left(C_{X}(X \times X)\right)$ the multiplicity of $C_{X}(X \times X)$ at $x$. In [4] we proved that $g(x)$ is the degree at $x$ of the Stückrad-Vogel cycle $v(X, X)=\sum_{C} j(X, X ; C)[C]$ of the self-intersection of $X$, that is, $g(x)=\sum_{C} j(X, X ; C) e_{x}(C)$ (see Proposition 3.6 for details). In this paper, our main result, Theorem 4.2, states that the pointwise degree $g(x)$ of the Stückrad-Vogel intersection cycle of the self-intersection of $X$ is a stratifying function which gives the canonical Whitney stratification. Our restriction to algebraic surfaces in $\mathbb{P}^{3}$ or $\mathbb{P}^{n}$ is only for simplicity. In fact, using Tworzewski's extension of the Stückrad-Vogel cycle to the analytic 
case (see $[\mathbf{1 8}]$ ), one has the analogous result for complex analytic surfaces in $\mathbb{C}^{n}$. Both in the projective and in the complex analytic case, the stratifying function $g(x)$ can be calculated by computer algebra systems - see our examples in $\S 4$.

Van Gastel conjectured that for hypersurfaces in $\mathbb{P}^{n}$ or $\mathbb{C}^{n}$, the pointwise degree $g(x)$ of the intersection cycle of a suitable multiple self-intersection should also give the canonical Whitney stratification. However, we were not able to prove that the $g$-constant strata are smooth. Hence we conclude our paper with some examples and open problems.

Notation 1.1. We denote the $n$-dimensional projective space over a field $K$ by $\mathbb{P}_{K}^{n}$. If not explicitly stated to the contrary, our base field will always be the field of complex numbers and we simply write $\mathbb{P}^{n}$. By a variety or subvariety of $\mathbb{P}_{K}^{n}$ we mean a closed reduced (but possibly reducible) equidimensional subscheme of $\mathbb{P}_{K}^{n}$ without embedded components. A surface is a 2-dimensional variety and a hypersurface an $(n-1)$-dimensional variety. The multiplicity of a scheme $X$ at a point $x$, i.e. the multiplicity of the local ring $\mathcal{O}_{X, x}$, will be denoted by $m_{x}(X)=e_{x}(X)=e\left(\mathcal{O}_{X, x}\right)$. Finally, $X_{\text {reg }}$ stands for the set of closed points $x$ of $X$ such that $\mathcal{O}_{X, x}$ is a regular local ring, and Sing $X$ is the complement of $X_{\text {reg }}$ in the set of closed points of $X$.

\section{Polar multiplicities and Whitney stratifications}

Definition 2.1. Let $X \subset \mathbb{P}^{n}$ be a $d$-dimensional subvariety, with $0 \leqslant d \leqslant n-1$, and for each $0 \leqslant k \leqslant d$ let $L_{(k)}$ be an $(n-d+k-2)$-dimensional linear subspace of $\mathbb{P}^{n}$. The polar variety (or polar locus) $P\left(L_{(k)}, X\right)$ of $X$ associated with $L_{(k)}$ is the closure of

$$
\left\{x \in X_{\text {reg }} \mid \operatorname{dim}\left(T_{x} X \cap L_{(k)}\right) \geqslant k-1\right\} .
$$

If $n-d+k-2=-1$ we set $P\left(L_{(k)}, X\right)=X$ and $L_{(k)}=\emptyset$. This happens only in the case $k=0$ and $d=n-1$.

Remark 2.2. If $L_{(k)}$ is a generic linear subspace of $\mathbb{P}^{n}$, then the polar variety $P\left(L_{(k)}, X\right)$ is either empty or equidimensional of codimension $k$ in $X$, and its degree does not depend upon $L_{(k)}$ (see, for example, [16, Proposition 1.2 and the Transversality Lemma 1.3]).

Notation 2.3. For $L_{(k)}$ generic we set $P_{k}(X):=P\left(L_{(k)}, X\right)$ and call it the general $k$-polar variety (or $k$-polar locus of $X$ ).

If

$$
\mathcal{L}: L_{(0)} \subset L_{(1)} \subset \cdots \subset L_{(d)}
$$

is a generic flag of linear subspaces of $\mathbb{P}^{n}$ with $\operatorname{dim} L_{(k)}=n-d+k-2$, then we have the following inclusion of the corresponding polar varieties

$$
X=P_{0}(X) \supset P_{1}(X) \supset \cdots \supset P_{d}(X) .
$$

If $x \in X$, we can consider the sequence of multiplicities

$$
M_{X, x}(\mathcal{L})=\left(m_{x}\left(P_{0}(X)\right), \ldots, m_{x}\left(P_{d-1}(X)\right)\right) .
$$


This sequence does not depend upon the choice of the general flag $\mathcal{L}$ (see [17, IV 3.1, p. 425]), and is in fact constant on a Zariski open subset of the variety of flags. The number $m_{x}\left(P_{k}(X)\right)$, for $k=0, \ldots, d-1$, is called the general $k$-polar multiplicity of $X$ at $x$. Note that $m_{x}\left(P_{d}(X)\right)=0$, since the germ of $P_{d}(X)$ at the point $x$ is empty (see [17, IV 3.3]).

Remark 2.4. The polar varieties $P_{k}(X)$ arise as sets of critical points of generic linear projections (see [13, (2.2.3), pp. 462, 463] or [17, p. 314]).

We recall the definition of a critical point of a differentiable map.

Definition 2.5. Let $f: M \rightarrow N$ be a differentiable map. A point $x \in M$ is called a critical point of $f$ if the rank of the tangent map

$$
f_{* x}: T_{x} M \rightarrow T_{f(x)} N
$$

is smaller than the maximal possible one, that is,

$$
\operatorname{rank} f_{* x}<\min \left(\operatorname{dim}_{x} M, \operatorname{dim}_{f(x)} N\right) .
$$

Remark 2.6. Maintaining the notation of Definition 2.1 and Remark 2.4, we observe that the $k$-polar variety $P\left(L_{(k)}, X\right)$ is the closure of the set of critical points of the restriction to $X$ of the linear projection on $\mathbb{P}^{d-k+1}$ with centre $L_{(k)}$. If $\operatorname{dim} L_{(k)}=-1$, that is, if $d=n-1$ and $k=0$, we can consider the affine cones $\hat{X}$ of $X$ and $\hat{L}_{(0)}=\{0\}$ of $L_{(0)}=\emptyset$ in $\mathbb{A}^{n+1}$ and take the linear projection $\hat{X} \backslash\{0\} \rightarrow \mathbb{A}^{n}$. The closure of the set of critical points of this map is $\hat{X}$; in this sense, $P_{0}(X)=X$ can also be regarded as a set of critical points.

Definition 2.7. Let $X$ be $d$-dimensional complex projective variety and let $Y$ be a non-singular subvariety of $X$. We say that the pair $\left(X_{\text {reg }}, Y\right)$ satisfies the Whitney conditions at a point $x_{0} \in Y$ if for each sequence $\left(x_{i}\right)$ of points of $X_{\text {reg }}$ and each sequence $\left(y_{i}\right)$ of points of $Y$ both converging to $x_{0}$ and such that the $\operatorname{limits}_{\lim _{x_{i} \rightarrow x_{0}}} T_{x_{i}} X$ and $\lim _{x_{i}, y_{i} \rightarrow x_{0}} \overline{x_{i} y_{i}}$ exist in the Grassmannians $G(d, n)$ and $G(1, n)$, respectively, one has

(a) $\lim _{x_{i} \rightarrow x_{0}} T_{x_{i}} X \supset T_{x_{0}} Y$; and

(b) $\lim _{x_{i} \rightarrow x_{0}} T_{x_{i}} X \supset \lim _{x_{i}, y_{i} \rightarrow x_{0}} \overline{x_{i} y_{i}}$.

We remark that (b) implies (a).

Theorem 2.8 (Teissier [17]). With the above notation, the pair $\left(X_{\mathrm{reg}}, Y\right)$ satisfies the Whitney conditions in $x_{0}$ if and only if the sequence of polar multiplicities

$$
m_{y}(X), m_{y}\left(P_{1}(X)\right), \ldots, m_{y}\left(P_{d-1}(X)\right)
$$

is locally constant in $Y$ around $x_{0}$.

Definition 2.9. With the above notation, a Whitney stratification of $X$ is given by a filtration of $X$ by algebraic sets $F_{i}$

$$
X=F_{0} \supseteq F_{1} \supseteq \cdots \supseteq F_{d+1}=\emptyset
$$

with the following properties. 
(i) For each $i=0, \ldots, d$, the locally closed subset $F_{i} \backslash F_{i+1}$ is either empty or is a nonsingular quasi-projective variety of pure codimension $i$. (The connected components of $F_{i} \backslash F_{i+1}(i=0, \ldots, d)$ are called the strata of the stratification.)

(ii) Whenever $S_{j}$ and $S_{k}$ are connected components of $F_{i} \backslash F_{i+1}$ and $F_{l} \backslash F_{l+1}$, respectively $(i, l=0, \ldots, d)$, with $S_{j} \subset \bar{S}_{k}$, then the pair $\left(S_{k}, S_{j}\right)$ satisfies the Whitney conditions (a) and (b).

Remark 2.10. In the case of a (reduced) surface $X \subset \mathbb{P}^{n}$, the above Theorem 2.8 of Teissier implies that a Whitney stratification of $X$ can be obtained as follows. Set $F_{0}=X$, $F_{1}=\operatorname{Sing} X$ (the singular locus of $X$ ), and, if $F_{1,1}, \ldots, F_{1, m}$ are the 1-dimensional irreducible components of $F_{1}$, set

$F_{2}=\operatorname{Sing} F_{1} \cup\left\{0\right.$-dimensional components of $\left.F_{1}\right\}$

$\cup \bigcup_{j=1}^{m}\left\{x \in F_{1, j} \mid\left(m_{x}(X), m_{x}\left(P_{1}(X)\right)\right)\right.$ is different from its generic value in $\left.F_{1, j}\right\}$

and $F_{3}=\emptyset$. This is the so-called canonical Whitney stratification, which is characterized by the fact that the connected components of the differences $F_{i} \backslash F_{i+1}$ are the strata of the minimal or coarsest Whitney stratification (see $[\mathbf{1 7}$, Chapter 6$]$ ).

\section{Intersection cycle of Stückrad and Vogel}

Let $X, Y$ be equidimensional closed subschemes of $\mathbb{P}_{K}^{n}=\operatorname{Proj}\left(K\left[X_{0}, \ldots, X_{n}\right]\right)$, where $K$ is an arbitrary field. For indeterminates $U_{i j}(0 \leqslant i, j \leqslant n)$ let $L$ be the pure transcendental field extension $K\left(U_{i j}\right)_{0 \leqslant i, j \leqslant n}$ and $X_{L}:=X \otimes_{K} L$, etc. Proving a Bezout Theorem for improper intersections, Stückrad and Vogel (see [8]) introduced a cycle $v(X, Y)=$ $v^{0}+\cdots+v^{n+1}$ on $X_{L} \cap Y_{L}$, which is obtained by an intersection algorithm on the ruled join variety

$$
J:=J\left(X_{L}, Y_{L}\right) \subset \mathbb{P}_{L}^{2 n+1}=\operatorname{Proj}\left(L\left[X_{0}, \ldots, X_{n}, Y_{0}, \ldots, Y_{n}\right]\right)
$$

as follows.

Let $\Delta$ be the 'diagonal' subspace of $\mathbb{P}_{L}^{2 n+1}$ given by the equations

$$
X_{0}-Y_{0}=\cdots=X_{n}-Y_{n}=0,
$$

let $H_{i} \subseteq J$ be the divisor given by the equation

$$
\ell_{i}:=\sum_{j=0}^{n} U_{i j}\left(X_{j}-Y_{j}\right)=0
$$

and set $\underline{\ell}:=\left(\ell_{0}, \ldots, \ell_{n}\right)$. Then one defines cycles $\beta^{k}$ and $v^{k}$ inductively by setting $\beta^{0}:=$ $[J]$. If $\beta^{k}$ is already defined, decompose the intersection

$$
\beta^{k} \cap H_{k}=v^{k+1}+\beta^{k+1} \quad(0 \leqslant k \leqslant \operatorname{dim} J),
$$


where the support of $v^{k+1}$ lies in $\Delta$ and where no component of $\beta^{k+1}$ is contained in $\Delta$. It follows that $v^{k}$ is a $(\operatorname{dim} J-k)$-cycle on $X_{L} \cap Y_{L} \cong J \cap \Delta$. The part of dimension $k$ of the cycle $v(X, Y):=v(\underline{\ell}, J):=\sum v^{k}$ will be denoted by $v_{k}$, so that the upper index denotes the codimension in the ruled join and the lower one the dimension of the cycle. In general, the cycle $v(X, Y)$ is defined over $L$. By a result of van Gastel [19, Proposition 3.9], a $K$-rational irreducible subvariety $C$ of $X_{L} \cap Y_{L}$ occurs in $v(X, Y)$ if and only if $C$ is a distinguished variety of the intersection of $X$ and $Y$ in the sense of Fulton [9, p. 95], and this is equivalent to the maximality of the analytic spread (see $[\mathbf{2}]$ ) or the maximality of the dimension of the so-called limit of join variety (see [7]).

Definition 3.1 (2.2.1 in [8]). The cycle $v(X, Y)$ is called the $v$-cycle of the intersection of $X$ and $Y$. An irreducible subvariety $C$ of $X_{L} \cap Y_{L}$ is said to be a characteristic subvariety if $C$ occurs in $v(X, Y)$. The coefficient of $C$ in $v(X, Y)$ is denoted by $j(X, Y ; C)$. Thus

$$
v(X, Y)=\sum_{C} j(X, Y ; C)[C],
$$

where $C$ runs through the characteristic subvarieties. The set of all these subvarieties is denoted by $\mathcal{C}=\mathcal{C}(X, Y)$. Moreover, the set of all elements of $\mathcal{C}$ which are defined over $K$ is denoted by $\mathcal{C}_{\text {rat }}=\mathcal{C}_{\text {rat }}(X, Y)$, that is, $\mathcal{C}_{\text {rat }}$ is the set of $K$-rational or distinguished or fixed subvarieties and $\mathcal{C} \backslash \mathcal{C}_{\text {rat }}$ is the set of the so-called non $K$-rational or movable subvarieties of the intersection of $X$ and $Y$.

Remark 3.2. The Stückrad-Vogel intersection cycle can also be constructed in the same way for more than two equidimensional closed subschemes $X_{1}, \ldots, X_{r} \subseteq \mathbb{P}_{K}^{n}$ by applying the intersection algorithm to the join variety $J\left(X_{1}, \ldots, X_{r}\right) \subseteq \mathbb{P}^{N}, N:=r(n+$ 1) -1 , and generic hyperplanes $H_{0}, \ldots, H_{N-n-1} \subset \mathbb{P}^{N}$ whose intersection is the diagonal $\Delta \subset \mathbb{P}^{N}$ (see, for example, [8, Remark 2.2.14]). The resulting cycle will be denoted by $v\left(X_{1}, \ldots, X_{r}\right)$.

We observe that for improper intersections the associativity law does not hold, that is, in general, $v\left(X_{1}, v\left(X_{2}, X_{3}\right)\right) \neq v\left(v\left(X_{1}, X_{2}\right), X_{3}\right)$ and both of these cycles may be also different from $v\left(X_{1}, X_{2}, X_{3}\right)$ (see [8, Example 2.2.15]). In $\S 4$ we will compare the cycles $v(X, X, X)$ and $v(v(X, X), X)$.

In the case of a self-intersection, we define inductively

$$
X^{(1)}:=X \quad \text { and } \quad X^{(m)}:=v\left(X^{(m-1)}, X\right),
$$

and we will see that on the smooth locus of $X$ this cycle is composed of polar varieties (see Proposition 3.5 below). In order to prove this proposition, we need the following definition and result from $[6]$.

Definition 3.3 (Definitions 3.5 and 4.1 in [6]). The map

$$
p:=\left(\ell_{0}: \cdots: \ell_{r}\right): \mathbb{P}_{L}^{n} \rightarrow \mathbb{P}_{L}^{r}
$$

is called the $r$ th generic linear projection. 
The set

$$
\operatorname{Sm}(X, Y):=\{x \in X \cap Y \mid X, Y \text { and } X \cap Y \text { are smooth at } x\}
$$

is called the smooth locus of the pair $(X, Y)$.

Theorem 3.4 (Theorem 4.6 in [6]). Let $X, Y \subseteq \mathbb{P}^{n}$ be algebraic varieties of dimension $d$ and $e$, respectively. Let $t$ be an integer such that $0 \leqslant t \leqslant \operatorname{dim} X \cap Y-1$ and $n \geqslant d+e-t-1$. Let $p: X_{L} \cup Y_{L} \rightarrow \mathbb{P}_{L}^{d+e-t-1}$ be the generic linear projection and $R(p)=R\left(p, X_{L}, Y_{L}\right)$ its ramification locus. Then $\operatorname{dim} R(p) \leqslant t$, and the associated $t$-cycle $[R(p)]_{t}$ is just $v_{t}(X, Y)$ on $\operatorname{Sm}\left(X_{L}, Y_{L}\right)$.

We observe that in a projective space over an infinite field $K$ the Stückrad-Vogel intersection cycle can be constructed also with elements $u_{i j}$ from $K$ instead of indeterminates $U_{i j}$. It is sufficient to choose these elements such that the linear forms $\ell_{i}$ avoid a finite number of certain prime ideals (see $[\mathbf{3}]$ for the precise conditions). The above results remain valid if we specialize the indeterminates $U_{i j}$ to generic elements $u_{i j}$ in $K$.

Proposition 3.5. Let $X \subset \mathbb{P}^{n}$ be a hypersurface (reduced and equidimensional without embedded components). Define inductively $X^{(1)}:=X$ and $X^{(m)}:=v\left(X^{(m-1)}, X\right)$. Then

$$
X^{(m)}=\sum_{k=0}^{m-1}\left(\begin{array}{c}
m-1 \\
k
\end{array}\right)\left[P_{k}(X)\right]+w_{m}, \quad 2 \leqslant m \leqslant n,
$$

where the support of $w_{m}$ is contained in the singular locus of $X$.

Proof. For $m=2$ the result follows immediately from Theorem 3.4, with $t=n-2$ and $d=e=n-1$, and Remark 2.4 with $k=1$ (since $\left[P_{0}(X)\right]=[X]$ ). By induction assume $m \geqslant 3$ and that

$$
X^{(m-1)}=\sum_{k=0}^{m-2}\left(\begin{array}{c}
m-2 \\
k
\end{array}\right)\left[P_{k}(X)\right]+w_{m-1} .
$$

Hence by the linearity of the Stückrad-Vogel cycle

$$
X^{(m)}=v\left(X^{(m-1)}, X\right)=\sum_{k=0}^{m-2}\left(\begin{array}{c}
m-2 \\
k
\end{array}\right) v\left(X, P_{k}(X)\right)+v\left(X, w_{m-1}\right) .
$$

Observe that the support of $v\left(X, w_{m-1}\right)$ is contained in the singular locus of $X$. Now, if $P_{k}(X) \neq \emptyset$,

$$
v\left(X, P_{k}(X)\right)=\left[P_{k}(X)\right]+v_{n-k-2}\left(X, P_{k}(X)\right)
$$

(see [8, Remark 2.2.7.(2)]). Moreover, again by Remark 2.4 and Theorem 3.4 in the nontrivial case where $P_{k}(X) \neq \emptyset$, with $t=n-k-2, d=n-1$ and $e=n-1-k$, noting that $P_{k}(X) \subseteq X$,

$$
v\left(X, P_{k}(X)\right)=\left[P_{k}(X)\right]+\left[P_{k+1}(X)\right]+\tilde{w}_{k},
$$


where $\tilde{w}_{k}$ is supported by the singular locus of $X$. Then we obtain

$$
X^{(m)}=\sum_{k=0}^{m-2}\left(\begin{array}{c}
m-2 \\
k
\end{array}\right)\left(\left[P_{k}(X)\right]+\left[P_{k+1}(X)\right]\right)+w_{m},
$$

and an easy computation gives the result.

For an arbitrary irreducible subvariety $Z \subseteq X_{L} \cap Y_{L} \subset \mathbb{P}_{L}^{n}$ we set $Z_{\Delta}:=J(Z, Z) \cap \Delta$. By $\hat{J}$ and $\hat{Z}_{\Delta}$ we denote the affine cones of the ruled join

$$
J:=J\left(X_{L}, Y_{L}\right) \subset \mathbb{P}_{L}^{2 n+1}
$$

and $Z_{\Delta}$ in the affine space $\mathbb{A}_{L}^{2 n+2}$. Let $(A, \mathfrak{m})$ be the local ring $\mathcal{O}_{\hat{J}, \hat{Z}_{\Delta}}$ and let $I \subset A$ be the ideal of the diagonal subspace $\Delta$ and let $G(X, Y ; Z)$ denote the associated graded ring

$$
G_{I}(A)=\bigoplus_{j=0}^{\infty} I^{j} / I^{j+1}
$$

If $Z$ is the empty subvariety of $\mathbb{P}^{n}$, then $A$ becomes the homogeneous ring of coordinates of the ruled join $J \subset \mathbb{P}_{L}^{2 n+1}$ localized at the irrelevant maximal ideal; that is, we obtain a global picture of the intersection algorithm. Let $e(B)$ denote the multiplicity of a local ring (or graded ring) $B$ with respect to its unique maximal (or homogeneous maximal) ideal. Finally, we denote by

$$
c_{k}:=c_{k}(I, A):=c_{k}\left(G_{\mathfrak{m}}\left(G_{I}(A)\right)\right) \quad(0 \leqslant k \leqslant \operatorname{dim} A=: d)
$$

the generalized Samuel multiplicities of the ideal $I \subset A$, which are defined by the leading coefficients of the Hilbert polynomial belonging to the twofold sum transform of the Hilbert function of the bigraded ring $R=\oplus_{i, j=0}^{\infty} R_{i j}$ with

$$
R_{i j}=G_{\mathfrak{m}}^{i}\left(G_{I}^{j}(A)\right)=\left(\mathfrak{m}^{i} I^{j}+I^{j+1}\right) /\left(\mathfrak{m}^{i+1} I^{j}+I^{j+1}\right)
$$

(see [4, Definition 2.2]).

Proposition 3.6 (Proposition 2.5, Corollary 4.2 and Corollary 4.4 in [4]). With the preceding notation,

$$
e(G(X, Y ; Z))=e\left(G_{I}(A)\right)=\sum_{C} j(X, Y ; C) e\left(\mathcal{O}_{C, Z}\right)=\sum_{k=0}^{d} c_{k}(I, A),
$$

where $C$ runs through the characteristic subvarieties of $X$ and $Y$ with $C \supseteq Z$.

If $Z=\emptyset$, then $d=\operatorname{dim} A=\operatorname{dim} J+1$,

$$
c_{0}=j(X, Y ; \emptyset), \quad c_{1}=\operatorname{deg} v_{0}, \quad c_{2}=\operatorname{deg} v_{1}, \quad \ldots, \quad c_{d}=\operatorname{deg} v_{d-1},
$$

and if $k>\operatorname{dim}(X \cap Y)+1$, then $c_{k}=0$. 
If $Z \neq \emptyset$ is $K$-rational, then $d=\operatorname{dim} A=\operatorname{dim} J-\operatorname{dim} Z$ and

$$
c_{k}=\sum_{C} j(X, Y ; C) e\left(\mathcal{O}_{C, Z}\right) \quad(0 \leqslant k \leqslant d),
$$

where $C$ runs through all varieties of $\mathcal{C}(X, Y)$ with $C \supseteq Z$ and $\operatorname{codim}_{C} Z=k$. If $k>$ $\operatorname{dim}(X \cap Y)-\operatorname{dim} Z$, then $c_{k}=0$.

Remark 3.7 (analytic case). In the paper [18], Tworzewski has constructed an intersection cycle of complex analytic subsets $X$ and $Y$ of a manifold $M$ which do not necessarily intersect properly. His construction is based on a pointwise defined intersection multiplicity $g(x)=g\left(X \times Y, \Delta_{M}, x\right)$ for a point $x \in \Delta_{M}$, where $\Delta_{M}$ is the diagonal of $M \times M$ and $g(x)$ is the sum of the coordinates of the so-called extended index of intersection $\tilde{g}(x)$ (see [18, Definition (4.2), p. 185]).

Let $A=\mathcal{O}_{X \times Y, x}$ and $I=\mathcal{I}_{\Delta_{M}} \mathcal{O}_{X \times Y, x}$. Nowak [14, 15] (see also [5]) has recently proved that $g(x)=e\left(G_{I}(A)\right)$ and that $\tilde{g}(X)$ is composed of the generalized Samuel multiplicities $c_{0}(I, A), \ldots, c_{\operatorname{dim}(X \cap Y)}(I, A)$ and of zeros.

In the analytic case, the Segre numbers of an ideal $I$ introduced by Gaffney and Gassler [10] are also special cases of the generalized Samuel multiplicities $c_{k}(I, A)$ (see [5]).

\section{Self-intersection and Whitney stratification}

If one wants to use the Stückrad-Vogel intersection cycle of a self-intersection of a surface $X$ in $\mathbb{P}^{3}$ for the construction of a Whitney stratification of $X$, then it seems to be natural to consider $v(X, X, X)$ or $v(v(X, X), X)$ rather than $v(X, X)$, since the latter cycle cannot have a 0-dimensional part (see [8, Remark 2.2.7.(2)]).

Van Gastel proposed to use $v(X, X, X)$ and conjectured that

$$
v(X, X, X)=v(v(X, X), X)
$$

(up to a field extension or up to rational equivalence). Such an associativity formula would then allow an application of Proposition 3.5. Unfortunately, the associativity law does not hold. Flenner suggested to us the following example from [8, Example 2.2.15] in order to show that in general $v(X, X, X) \neq v(v(X, X), X)$.

Example 4.1. Consider the configuration of two lines $V_{1}, V_{2}$ and of a conic $V_{3}$ in the projective plane (Figure 1).

Let $X_{i}$ be the cone over $V_{i}$ with common vertex $C$ in $\mathbb{P}^{3}$ and denote by $L_{P}, L_{Q}$ the lines $P C$ and $Q C$, respectively. We want to calculate the twofold self-intersections of the surface

$$
X:=X_{1} \cup X_{2} \cup X_{3} \subset \mathbb{P}^{3}=\operatorname{Proj}(\mathbb{C}[x, y, z, w]),
$$

which is given by the equation $x y\left(y z-x^{2}\right)=0$. Obviously,

$$
\begin{gathered}
v\left(X_{1}, X_{1}\right)=X_{1}, \quad v\left(X_{1}, X_{2}\right)=L_{P}, \quad v\left(X_{2}, X_{2}\right)=X_{2}, \\
v\left(X_{2}, X_{3}\right)=L_{P}+L_{Q} \quad \text { and } \quad v\left(X_{3}, X_{3}\right)=X_{3}+L_{1}+L_{2},
\end{gathered}
$$




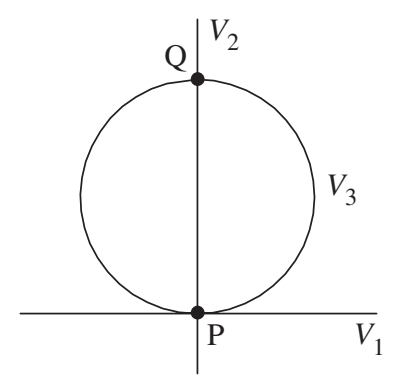

Figure 1. Comparison of threefold self-intersections.

where $L_{1}$ and $L_{2}$ are movable lines on the cone $X_{3}$ passing through the vertex $C$. Hence

$$
v\left(X_{1}, L_{i}\right)=C, \quad v\left(X_{2}, L_{i}\right)=C, \quad v\left(X_{3}, L_{i}\right)=L_{i}+C .
$$

Furthermore,

$$
\begin{array}{lll}
v\left(X_{1}, L_{P}\right)=L_{P}, & v\left(X_{1}, L_{Q}\right)=C, & v\left(X_{2}, L_{P}\right)=L_{P}, \\
v\left(X_{2}, L_{Q}\right)=L_{Q}, & v\left(X_{3}, L_{P}\right)=L_{P}+C, & v\left(X_{3}, L_{Q}\right)=L_{Q}+C .
\end{array}
$$

Using the bilinearity of the Stückrad-Vogel cycle we get

$$
v(v(X, X), X)=X_{1}+X_{2}+X_{3}+32 L_{P}+6 L_{Q}+L_{1}+\cdots+L_{4}+18 C,
$$

where $L_{3}$ and $L_{4}$ are two further movable lines on $X_{3}$ going through $C$. The calculation of $v(X, X, X)$ is more difficult. Again by the bilinearity of the cycle, we have to sum up 27 cycles $v_{i j k}:=v\left(X_{i}, X_{j}, X_{k}\right)$, which by symmetry can be grouped as follows:

$$
\begin{aligned}
& v_{123}=v_{132}=v_{213}=v_{231}=v_{312}=v_{321}=L_{P}+C, \\
& v_{112}=v_{121}=v_{211}=v_{122}=v_{212}=v_{221}=L_{P}, \\
& v_{113}=v_{131}=v_{311}=2 L_{P}, \\
& v_{133}=v_{313}=v_{331}=2 L_{P}+2 C, \\
& v_{223}=v_{232}=v_{322}=L_{P}+L_{Q}, \\
& v_{233}=v_{323}=v_{332}=L_{P}+L_{Q}+2 C \\
& v_{111}=X_{1} \text { and } v_{222}=X_{2}, \\
& v_{333}=X_{3}+L_{1}+\cdots+L_{4}+2 C,
\end{aligned}
$$

where $L_{1}, \ldots, L_{4}$ are again movable lines as before. It follows that

$$
v(X, X, X)=X_{1}+X_{2}+X_{3}+30 L_{P}+6 L_{Q}+L_{1}+\cdots+L_{4}+20 C .
$$

This time the contribution of the vertex $C$ is higher by 2 , but that of the line $L_{P}$ smaller by 2 so that we have of course the same Bezout number 64 . 
Surprisingly, it turns out that neither $v(X, X, X)$ nor $v(v(X, X), X)$ is needed in order to get a Whitney stratification of the surface $X$. As we will see in the next theorem, it is sufficient to consider the self-intersection $v(X, X)$ and to take its local degree $g(x)$ as a stratifying function.

Theorem 4.2. Let $X \subset \mathbb{P}^{n}$ be a (reduced) surface, $x \in X$ be a closed point and $g: X \rightarrow \mathbb{N}$ be the map defined by $g(x)=e(G(X, X ; x))$. Then

$$
X_{j}:=\{x \in X \mid g(x) \geqslant j\}, \quad j=0,1, \ldots,
$$

are closed subschemes of $X$ or empty, and the connected components of

$$
S_{g}(j):=g^{-1}(j)=X_{j} \backslash X_{j+1}
$$

are the strata of a Whitney stratification of $X$ (the coarsest one if $n=3$ ).

Proof. At first we will show that $X_{j}$ is closed. In fact, given a closed point $x \in X \backslash X_{j}$, we will construct a Zariski open subset $U$ of $X$ containing $x$ such that $g(y) \leqslant g(x)$ for each closed point $y \in U$. Let

$$
\mathcal{C}(X, X)=\left\{C_{1}, \ldots, C_{s}, D_{s+1}, \ldots, D_{t}\right\}
$$

be the set of the characteristic subvarieties of the self-intersection of $X$, and assume that $C_{1}, \ldots, C_{s}$ pass through $x$ but $D_{s+1}, \ldots, D_{t}$ do not. By [12, Lemma 2.2] the sets

$$
D_{i}:=\left\{y \in C_{i} \mid e\left(\mathcal{O}_{C_{i}, y}\right)>e\left(\mathcal{O}_{C_{i}, x}\right)\right\}, \quad i=1, \ldots, s,
$$

are closed in $C_{i}$ and hence in $X$. Then

$$
U:=X \backslash\left(\bigcup_{i=1}^{t} D_{i}\right)
$$

is a Zariski open subset of $X$ containing $x$ such that

$$
g(y)=\sum_{i=1}^{s} j\left(X, X ; C_{i}\right) e\left(\mathcal{O}_{C_{i}, y}\right) \leqslant g(x)=\sum_{i=1}^{s} j\left(X, X ; C_{i}\right) e\left(\mathcal{O}_{C_{i}, x}\right)
$$

for all $y \in U$.

Now we prove that $S_{g}(j)$ is smooth.

At first we note that $g(x)=1$ if and only if $X$ is smooth at $x$. In fact, $g(x)=1$ forces $X$ to be irreducible at $x$. Thus $g(x)=m_{x}(X)+\cdots=1$, which implies $m_{x}(X)=1$. Conversely, the regularity of $\mathcal{O}_{X, x}$ implies the regularity of $A=\mathcal{O}_{\hat{J}, \hat{x}_{\Delta}}$ (see, for example, [8, Corollary 1.3.15]) and of $A / I \cong \mathcal{O}_{\hat{X}, \hat{x}}$, where we used our notation introduced before Proposition 3.6. Hence $I$ is generated by regular parameters, $G_{I}(A)$ is a polynomial ring over the regular local ring $A / I$, and $g(x)=e\left(G_{I}(A)\right)=1$.

If $\operatorname{dim}(\operatorname{Sing} X)=0$, then, by the above consideration, the stratification is given by $X$ and by the singular points of $X$. So the strata are smooth. 
If $\operatorname{dim}(\operatorname{Sing} X)=1$, then

$$
v(X, X)=[X]+\left[P_{1}(X)\right]+\sum_{k=1}^{t} j_{k}\left[C_{k}\right]+\text { eventual 0-dimensional part }
$$

where the $C_{k}(k=1, \ldots, t)$ are all the 1-dimensional irreducible components of the singular locus of $X$ counted by their intersection numbers $j_{k}$ (see Proposition 3.5 and $[\mathbf{2}$, Corollary 2.5]). The 0-dimensional part can only occur if $n \geqslant 4$ (see [8, Remark 2.2.7.(2)]), and its fixed part (if there is any) is supported on Sing $X$ (see [2, Corollary 2.5]) and its movable part is $\left[P_{2}(X)\right]$. Note that for a point $x \in X, g(x)$ is the degree of $v(X, X)$ at $x$ (Proposition 3.6):

$$
g(x)=m_{x}(X)+m_{x}\left(P_{1}(X)\right)+\sum_{k=1}^{t} j_{k} m_{x}\left(C_{k}\right)+j(X, X ; x) .
$$

Here we have used that $m_{x}\left(P_{2}(X)\right)=0$ (see the remark at the end of Notation 2.3). Now consider a point $x$ on a fixed $C_{k}$, and call it a general point of $C_{k}$ if the following four conditions are satisfied.

(i) $x$ is a smooth point of $C_{k}$ (equivalently, $m_{x}\left(C_{k}\right)=1$ ) and $m_{x}(X)=e\left(\mathcal{O}_{X, C_{k}}\right)$.

(ii) $x \notin P_{1}(X)$; that is, $m_{x}\left(P_{1}(X)\right)=0$.

(iii) $x$ does not belong to $C_{i}$ with $i \neq k$; that is, $m_{x}\left(C_{i}\right)=0$ if $i \neq k$.

(iv) $x$ does not belong to the 0 -dimensional part of $v(X, X)$; that is, $j(X, X ; x)=0$.

In all general points of $C_{k}$, the function $g$ takes the same value $a:=e\left(\mathcal{O}_{X, C_{k}}\right)+j_{k}$, but $g(x)$ will be strictly larger than $a$ if $x \in C_{k}$ is not a general point of $C_{k}$. This can be easily seen from the above description of $g(x)$. For example, if $x$ is a singular point of $C_{k}$, then $m_{x}\left(C_{k}\right)=e\left(\mathcal{O}_{C_{k}, x}\right)>1$. It follows that the 1-dimensional strata are formed by the general points of $C_{k}(k=1, \ldots, t)$ and that they are smooth. As in the case $\operatorname{dim}(\operatorname{Sing} X)=0$, every isolated singular point of $X$ (which could also be in $v(X, X)$ if $n \geqslant 4$ ) itself forms a stratum. The 2 -dimensional strata, being the connected components of $X \backslash$ Sing $X$, are obviously smooth.

Now we know that $S_{g}(j):=g^{-1}(j)=X_{j} \backslash X_{j+1}$ is smooth and that, by construction, for all $x \in X_{j} \backslash X_{j+1}$ it holds that, following the notation of Proposition 3.5,

$$
g(x)=m_{x}(X)+m_{x}\left(P_{1}(X)\right)+m_{x}(w)=j .
$$

Here $w$ denotes the part of $v(X, X)$ whose support is contained in the singular locus of $X$, and we have used that, as noted previously, $m_{x}\left(P_{2}(X)\right)=0$ (which is relevant only if $n \geqslant$ 4). By the upper-semicontinuity of the multiplicities $m_{x}(\cdots)$, it follows that both $m_{x}(X)$ and $m_{x}\left(P_{1}(X)\right)$ must be constant on the connected components of $S_{g}(j)$ and hence, by Teissier's result (Theorem 2.8), the connected components of $S_{g}(j)$ are the strata of a Whitney stratification of $X$. Moreover, if $n=3$, we have then obtained the coarsest 


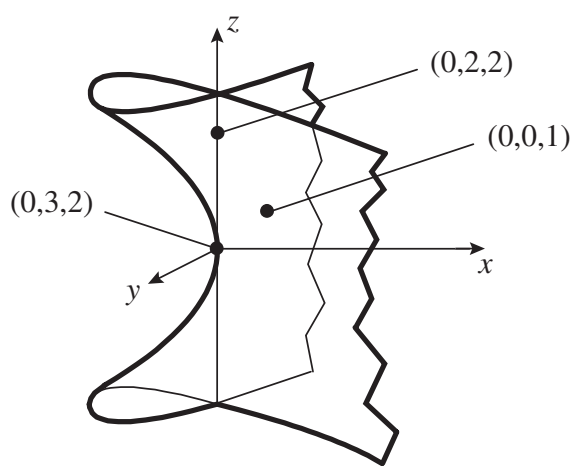

Figure 2. The $g$-stratification of the Whitney umbrella.

Whitney stratification of $X$. In fact, to get smooth strata, $\sum_{k=1}^{t} j_{k} m_{x}\left(C_{k}\right)=m_{x}(w)$ (for this equality we have used that $n=3$ ) must be constant along each stratum of an arbitrary Whitney stratification and hence, again by Theorem $2.8, g(x)$ must be constant, which is the condition used to construct our stratification. This finishes the proof.

One is tempted to ask whether a Whitney stratification of a surface $X$ can be obtained by other stratifying functions coming from self-intersections. Of course, by Remark 3.2, one could also consider the cycles $v(X, X, X)$ and $v(v(X, X), X)$ and take again their pointwise degree as stratifying functions to obtain what we call the $g$-stratifications of $X$ by $v(X, X, X)$ and $v(v(X, X), X)$, respectively. Or one could stratify $X$ by the pointwise degree of the fixed part only of $v(X, X), v(X, X, X)$ and $v(v(X, X), X)$, getting stratifications which we call the distinguished stratifications of $X$ by $v(X, X), v(X, X, X)$ and $v(v(X, X), X)$, respectively.

The complete comparison of the $g$-stratifications and of the distinguished stratifications by $v(X, X), v(X, X, X)$ and $v(v(X, X), X)$ is still open. In particular, it is not known if the distinguished stratification by $v(X, X, X)$ coincides with the $g$-stratification by $v(X, X, X)$, but an example from Teissier [17, p. 315] shows that for $v(X, X)$ the two stratifications may differ and that the distinguished one does not need to be a Whitney stratification.

Example 4.3 (Teissier). Consider the hypersurface $X \subset \mathbb{C}^{3}$ with the defining equation $y^{2}-x^{3}-z^{2} x^{2}=0$ : see Figure 2 .

The singular locus of $X$ is the $z$-axis, which is the only distinguished subvariety (except the whole surface $X$ ). So the distinguished stratification has only two strata and hence is not a Whitney stratification (see $[\mathbf{1 7}$, p. 315]). In order to find the $g$-stratification of the preceding theorem, we calculate the generalized Samuel multiplicities

$$
\left(c_{0}(I, A), c_{1}(I, A), c_{2}(I, A)\right)
$$

for $A:=\mathcal{O}_{X \times X, a}(a \in X$, and $X$ is considered to be diagonally embedded in $X \times X)$ and $I=\mathcal{I}_{\Delta} A$. This can be done by using the computer program CALI [11], a REDUCE package for commutative algebra, together with the script [1] written by Aliffi and the 
first author. We obtain $(0,3,2)$ if the point $a \in X$ is the origin, $(0,2,2)$ for $a$ on the $z$-axis but different from the origin, and $(0,0,1)$ for all smooth points $a$ of $X$ (see Figure 2). Note that $g(a)=c_{0}(I, A)+c_{1}(I, A)+c_{2}(I, A)$. Therefore, we have three strata: the origin with $g=5$ (which is not a stratum in the distinguished stratification); the $z$-axis except the origin with $g=4$; and $X_{\text {reg }}$ with $g=1$.

In a similar way one finds the canonical Whitney stratification of the projective closure $\bar{X}$ of $X$ in $\mathbb{P}^{3}=\operatorname{Proj}(\mathbb{C}[t, x, y, z])$. The singular locus of $\bar{X}$ consists of the two lines $L_{1}: x=y=0$ and $L_{2}: t=x=0$, which intersect at the point $P_{3}=(0,0,0,1)$. To construct a Whitney stratification, the points $P_{1}=(1,0,0,0)$ and $P_{2}=(0,0,1,0)$ are also needed. The canonical Whitney stratification of $\bar{X}$ has the following six strata: $\bar{X}_{\text {reg }}$ with $g=1, L_{1} \backslash\left(P_{1} \cup P_{3}\right)$ and $L_{2} \backslash\left(P_{2} \cup P_{3}\right)$ with $g=4$, the point $P_{1}$ with $g=5$, and the points $P_{3}$ and $P_{2}$ with $g=6$.

In the following example we study a surface $X$ whose $g$-stratification by $v(X, X)$ is different from its distinguished stratification by $v(v(X, X), X)$.

Example 4.4. Let $X \subset \mathbb{C}^{3}$ be the union of the plane $X_{1}$ with defining equation $z=0$ and the non-singular surface $X_{2}$ with defining equation $z-x^{3}+y^{2}=0$. Then the singular locus of $X$ is the cuspidal plane curve $C:=X_{1} \cap X_{2}$ with cusp point $O$. By the bilinearity of the Stückrad-Vogel cycle we get easily $v(X, X)=X_{1}+X_{2}+2 C+P$, where $P$ is a (movable) polar curve of $X_{2}$ that can be moved away from $O$. Using again the bilinearity we find $v(X, v(X, X))$, and since $O$ is a smooth point both of $X_{1}$ and $X_{2}$ and is not lying on a general polar curve $P$, the point $O$ will not appear in the cycle $v(X, v(X, X))$. But the point $O$ is a singular point of $C=\operatorname{Sing} X$ and hence the function $g(x)=m_{x}\left(X_{1}\right)+m_{x}\left(X_{2}\right)+2 m_{x}(C)$ (see $v(X, X)$ above), being 1 on $X \backslash C$ and 4 on $C \backslash O$, has in $O$ the value $1+1+2 m_{x}(C)=6$.

Let $X$ be a hypersurface in $\mathbb{P}^{n}$ and consider the $g$-stratifications of $X$ coming from $X^{(n-1)}$ or $X^{(n)}$, respectively. If the $g$-constant strata were smooth, then one could generalize Theorem 4.2 to $X \subset \mathbb{P}^{n}, n \geqslant 4$. Unfortunately, this is not the case, at least for reducible hypersurfaces. We have the following counterexample.

Example 4.5. Consider the projective closure in $\mathbb{P}^{4}$ of the hypersurface $X \subset \mathbb{C}^{3}$ which is defined as the union of the two smooth hypersurfaces $X_{1}: t=0$ and $X_{2}: t+z\left(z+y^{2}-\right.$ $\left.x^{3}\right)=0$. The singular locus of $X$ is $X_{1} \cap X_{2}$ and consists of the plane $S_{1}: t=z=0$ and the smooth surface $S_{2}: t=z+y^{2}-x^{3}=0$. Note that $C:=\operatorname{Sing}(\operatorname{Sing} X)=S_{1} \cap S_{2}$ is a plane curve with a cusp at the origin. We will see that the stratifying function $g$ is constant on $C$ and does not jump at the cusp point. To this end we calculate the Stückrad-Vogel cycle that defines $g$. Obviously, $v\left(X_{1}, X_{1}\right)=X_{1}, v\left(X_{2}, X_{2}\right)=X_{2}, v\left(X_{1}, X_{2}\right)=S_{1}+S_{2}$, and using the bilinearity of the Stückrad-Vogel cycle we get

$$
v(X, X)=X_{1}+X_{2}+2\left(S_{1}+S_{2}\right), \quad X^{(3)}=v(v(X, X), X)=X_{1}+X_{2}+6\left(S_{1}+S_{2}\right)
$$

and

$$
X^{(4)}=v\left(X^{(3)}, X\right)=X_{1}+X_{2}+14\left(S_{1}+S_{2}\right) .
$$

This implies that $g$ is constant on $C$, namely 14 if $g$ is defined by $X^{(3)}$ or 30 if it is defined by $X^{(4)}$. 
We conclude with two open problems.

Problem 4.6. Let $X$ be an irreducible (and reduced) hypersurface in $\mathbb{P}^{n}$ and consider the $g$-stratification of $X$ by $X^{(n)}$. Are the $g$-constant strata smooth? A positive answer would allow us to generalize Theorem 4.2 to such $X \subset \mathbb{P}^{n}$-but see the preceding counterexample if $X$ is not irreducible.

Problem 4.7. Let $X$ be a (reduced) surface in $\mathbb{P}^{3}$. Is the distinguished stratification by $v(X, X, X)$ equal to the $g$-stratification by $v(X, X)$, that is, to the canonical Whitney stratification of $X$ ?

Acknowledgements. The investigations in this paper were supported by the MURST and the University of Bologna, Funds for Selected Research Topics. The authors are members of the GNSAGA of the Italian CNR.

It is a great pleasure for the authors to thank Leendert van Gastel, who generously discussed his conjectures and ideas of possible proofs with them. The authors are also grateful to the referee for useful suggestions on how to improve this paper.

\section{References}

1. R. Achilles and D. Aliffi, Segre: a script for the Reduce package Cali (Bologna, 1999-2001), http://www.dm.unibo.it/ achilles/segre/.

2. R. AChilles And M. Manaresi, An algebraic characterization of distinguished varieties of intersection, Rev. Roumaine Math. Pures Appl. 38 (1993), 569-578.

3. R. Achilles And M. Manaresi, Multiplicity for ideals of maximal analytic spread and intersection theory, J. Math. Kyoto Univ. 33 (1993), 1029-1046.

4. R. Achilles and M. Manaresi, Multiplicities of a bigraded ring and intersection theory, Math. Ann. 309 (1997), 573-591.

5. R. Achilles And S. Rams, Intersection numbers, Segre numbers and generalized Samuel multiplicities, Arch. Math. 77 (2001), 391-398.

6. H. FlenNER AND M. MANARESI, Intersections of projective varieties and generic projections, Manuscr. Math. 92 (1997), 273-286.

7. H. Flenner and W. Vogel, Limits of joins and intersections, in Higher dimensional complex varieties, Proc. Int. Conf., Trento (1994), pp. 209-220 (Walter de Gruyter, Berlin, 1996).

8. H. Flenner, L. O'Carroll and W. Vogel, Joins and intersections, Springer Monographs in Mathematics (Springer, 1999).

9. W. Fulton, Intersection theory, Ergebnisse der Mathematik und ihrer Grenzgebiete, 3 Folge, Bd. 2 (Springer, 1984).

10. T. Gaffney and R. Gassler, Segre numbers and hypersurface singularities, J. Alg. Geom. 8 (1999), 695-736.

11. H.-G. GRÄBE, CALI-a REdUCE package for commutative algebra, Version 2.2 .1 (1995), available through the REDUCE library (email: redlib@rand.org).

12. M. IDÀ AND M. MANARESI, Some remarks on normal flatness and multiplicity in complex spaces, in Commutative algebra, Trento, 1981, pp. 171-182, Lecture Notes in Pure and Applied Mathematics, vol. 84 (Dekker, New York, 1983).

13. LÊ DũNg Tráng And B. Teissier, Variétés polaires locales et classes de Chern des variétés singulières, Ann. Math. 114 (1981), 457-491.

14. K. NowaK, Analytic improper intersections, I, Deformation to the normal cone, Bull. Polish Acad. Sci. Math. 48 (2000), 121-130. 
15. K. NowAK, Analytic improper intersections, II, Deformation to an algebraic bicone and applications, Bull. Polish Acad. Sci. Math. 48 (2000), 131-140.

16. R. Piene, Polar classes of singular varieties, Annls Sci. Ec. Norm. Super. 11 (1978), 247-276.

17. B. TeIssier, Variétés polaires, II, Multiplicités polaires, sections planes, et conditions de Whitney, in Proc. La Rábida, 1981 (ed. J. M. Aroca, R. Buchweitz, M. Giusti and M. Merle), Springer Lecture Notes in Mathematics, vol. 961, pp. 314-491 (Springer, 1982).

18. P. Tworzewski, Intersection theory in complex analytic geometry, Ann. Polon. Math. 62 (1995), 177-191.

19. L. J. VAn GASTEL, Excess intersections and a correspondence principle, Invent. Math. 103 (1991), 197-222.

20. H. Whitney, Tangents to an analytic variety, Ann. Math. 81 (1964), 496-549. 